\title{
Investigation into the Pressure-Driven Extension of the EPANET Hydraulic Simulation Model for Water Distribution Systems
}

\author{
Alemtsehay G. Seyoum ${ }^{1}$ - Tiku T. Tanyimboh ${ }^{1}$
}

Received: 10 December 2015 / Accepted: 5 September 2016/

Published online: 29 September 2016

(C) The Author(s) 2016. This article is published with open access at Springerlink.com

\begin{abstract}
Several hydraulic modelling approaches have been proposed previously to simulate pressure - deficient operating conditions in water distribution networks more realistically. EPANETPDX is a pressure-driven extension of the EPANET 2 hydraulic simulation model that has an embedded logistic nodal head-flow function. The pressure-driven analysis algorithm in EPANETPDX was investigated, to improve its performance under conditions of extremely low pressure. By integrating a line minimization procedure fully in the computational solution of the system of equations, the algorithm's consistency was improved by increasing its computational efficiency under conditions of extremely low pressure. The examples considered demonstrated that the pressure-driven analysis algorithm proposed is robust, computationally efficient, and the line minimization procedure is applied frequently. Overall, the results suggest that the algorithm is reliable. The formulation proposed is significantly faster than the previous model under conditions of extremely low pressure. The hydraulic and water quality modelling functionality of EPANET 2 was preserved. For the operating conditions with satisfactory pressure, where direct comparisons with EPANET 2 were possible, EPANET 2 was consistently faster.
\end{abstract}

Keywords Nodal head-flow function - Water distribution network - Line minimization · Evolutionary optimization · Global gradient algorithm $\cdot$ Pressure-driven analysis

Electronic supplementary material The online version of this article (doi:10.1007/s11269-016-1492-6) contains supplementary material, which is available to authorized users.

Tiku T. Tanyimboh

tiku.tanyimboh@strath.ac.uk

1 Department of Civil and Environmental Engineering, University of Strathclyde, James Weir Building, 75 Montrose Street, Glasgow, G1 1XJ, UK 


\section{Introduction}

Hydraulic simulation models of water distribution networks are invaluable for design and operation purposes. They help predict the properties of the flow including water quality under normal and abnormal operating conditions. Moreover, there is increasing emphasis on the application of hydraulic simulation models for solving optimisation problems by combining them with evolutionary algorithms (Kougias and Theodossiou 2013; Méndez et al. 2013; Dini and Tabesh 2014; Bragalli et al. 2016). The simulation models evaluate the equations for conservation of mass and energy and other properties such as nodal pressures (SeifollahiAghmiuni et al. 2013; Kang and Lansey 2014; Tao et al. 2014; Yang and Boccelli 2014; Laucelli and Giustolisi 2015; Kun et al. 2015). Evolutionary algorithms, by nature, often generate numerous infeasible candidate solutions. While conventional demand-driven hydraulic simulation models (Rossman 2000; Spiliotis and Tsakiris 2011) are incapable of simulating pressure-deficient conditions or infeasible solutions satisfactorily, pressure-driven models (Gorev and Kodzhespirova 2013; Tsakiris and Spiliotis 2014) do so realistically.

Evolutionary algorithms are essentially unconstrained optimisation procedures. On the other hand, many real world optimisation problems have multiple constraints and, consequently, the need for satisfactory approaches for incorporating constraints arises. Previous studies have demonstrated the benefits of explicitly maintaining infeasible solutions among the candidate solutions for single- and multi-objective constrained optimization problems (Singh et al. 2008; Ray et al. 2009). For example, some recent evolutionary optimization algorithms that retain infeasible solutions until the last generation achieved good results in terms of the convergence rate and quality of solutions (Siew and Tanyimboh 2012b; Saleh and Tanyimboh 2013, 2014).

Considering the optimization of real-world networks with hundreds or thousands of pipes, where the computational time for evaluating millions of candidate solutions to carry out multiple runs of an evolutionary algorithm could be prohibitive, a computationally efficient and robust pressure-driven simulation algorithm is vital. Very recently, Elhay et al. (2015) emphasized the urgent need for hydraulic models that are suitable for extreme operating conditions and/or extreme events e.g. electrical power failure and terrorist attacks.

The pressure-driven simulation algorithm in this article builds on the pressure-dependent extension of the EPANET hydraulic simulator, i.e. EPANET-PDX (Siew and Tanyimboh 2012a). EPANET-PDX has an integrated logistic nodal head-flow function (Tanyimboh and Templeman 2010) plus a line minimization procedure that facilitates convergence in the computational solution of the constitutive equations. This paper describes the EPANET-PDX algorithm in detail and addresses weaknesses uncovered under conditions of extremely low pressure (Seyoum 2015).

The modelling functionality of EPANET 2 (Rossman 2000) was preserved in full including extended period simulation and water quality modelling (Seyoum and Tanyimboh 2014). By contrast, some investigations into pressure-driven analysis (e.g. Kovalenko et al. 2014; Elhay et al. 2015) did not consider extended period simulation. Furthermore, they excluded key elements e.g. tanks, pumps and control devices. Control devices (e.g. pressure regulating valves) make the constitutive equations for water distribution systems difficult to solve due to convergence problems (Rossman 2007; Deuerlein et al. 2009; Kovalenko et al. 2014; Elhay et al. 2015). While Tanyimboh and colleagues (Tanyimboh et al. 2003; Tanyimboh and Templeman 2010) included pumps and control devices, extended period simulation was not considered. An extensive review of pressure-driven simulation and its applications is available in Abdy Sayyed et al. (2015). Other recent works on head-driven simulation include Elhay 
et al. (2015) and Sivakumar and Prasad (2015). Reviews of nodal head-flow relationships are available in Ciaponi et al. (2015) and Vairagade et al. (2015).

\section{Formulation of The Pressure-Driven Simulation Model}

The EPANET 2 hydraulic simulator (Rossman 2000) uses the global gradient algorithm (Todini and Pilati 1988) to solve the constitutive equations. Siew and Tanyimboh (2012a) extended the algorithm by enabling it to simulate pressure-deficient conditions realistically. The pressure dependent model known as EPANET-PDX integrates the continuous nodal head-flow function that Tanyimboh and Templeman (2010) proposed in the constitutive equations. The nodal head-flow function is

$$
Q n_{i}\left(H n_{i}\right)=Q n_{i}^{r e q} \frac{\exp \left(\alpha_{i}+\beta_{i} H n_{i}\right)}{1+\exp \left(\alpha_{i}+\beta_{i} H n_{i}\right)}
$$

Its first derivative is

$$
\frac{d Q n_{i}\left(H n_{i}\right)}{d H n_{i}}=Q n_{i}^{r e q} \beta_{i} \frac{\exp \left(\alpha_{i}+\beta_{i} H n_{i}\right)}{\left(1+\exp \left(\alpha_{i}+\beta_{i} H n_{i}\right)\right)^{2}}
$$

where, for node $i, Q n_{i}$ and $H n_{i}$ are the flow and head, respectively; $Q n_{i}^{r e q}$ is the demand; $\alpha_{i}$ and $\beta_{i}$ are parameters that are determined by calibration with field data. A Monte Carlo simulation procedure for calibrating $\alpha_{i}$ and $\beta_{i}$ is available in Ciaponi et al. (2015).

Tanyimboh and Templeman (2010) provided a generic procedure for estimating $\alpha_{i}$ and $\beta_{i}$ that may be summarized briefly as follows.

$$
\begin{gathered}
Q n_{i}\left(H n_{i}^{\mathrm{min}}\right)=\varepsilon_{1} Q n_{i}^{r e q} \\
Q n_{i}\left(H n_{i}^{r e q}\right)=\left(1-\varepsilon_{2}\right) Q n_{i}^{r e q}
\end{gathered}
$$

where $H n_{i}^{\text {min }}$ and $H n_{i}^{\text {req }}$ are, respectively, the head below which flow at node $i$ is unacceptably low or effectively zero, and above which the demand is satisfied in full effectively. $\varepsilon_{1}$ and $\varepsilon_{2}$ represent small tolerances that may be selected to suit the circumstances, for example, say $\varepsilon_{1}=$ 0.01 and $\varepsilon_{2}=0.001$ (as in Tanyimboh and Templeman 2010). Kovalenko et al. (2014) adopted $\varepsilon_{1}=\varepsilon_{2}=0.001$. Self-evidently the two values need not be identical, and any suitable combination may be used. Eq. 3 implies that the available flow would be practically zero at $H n_{i} \approx$ $H n_{i}^{\text {min }}$. Similarly, Eq. 4 implies that the available flow would be practically satisfactory at $H n_{i} \approx H n_{i}^{r e q}$. Illustrative examples are available in Ciaponi et al. (2015).

Eq. 1 represents a family of curves, and $\varepsilon_{1}$ and $\varepsilon_{2}$ lead to values of $\alpha_{i}$ and $\beta_{i}$ that adjust the shape of the nodal head-flow function in Eq. 1. Approximate values of $\alpha_{i}$ and $\beta_{i}$ may be obtained by substituting the values of $H n_{i}{ }^{\text {min }}, H n_{i}^{\text {req }}, \varepsilon_{1}$ and $\varepsilon_{2}$ in Eqs. 3 and 4 the solution of which provides the values of $\alpha_{i}$ and $\beta_{i}$. Following Tanyimboh and Templeman (2010), $\varepsilon_{1}$ and $\varepsilon_{2}$ were taken as 0.01 and 0.001 , respectively.

The system of equations applied in the global gradient algorithm is

$$
\left[\begin{array}{ccc}
\boldsymbol{A}_{11} & \vdots & \boldsymbol{A}_{12} \\
\cdots & \cdots & \cdots \\
\boldsymbol{A}_{21} & \vdots & 0
\end{array}\right]\left[\begin{array}{c}
\boldsymbol{Q p} \\
\cdots \\
\boldsymbol{H n}
\end{array}\right]=\left[\begin{array}{c}
-\boldsymbol{A}_{10} \boldsymbol{H}_{0} \\
\cdots \\
-\boldsymbol{Q n}^{r e q}
\end{array}\right]
$$


$\boldsymbol{A}_{11}$ is a diagonal matrix whose elements are $K_{i j}\left(Q p_{i j}\right)^{n_{f}-1} ; K_{i j}$ and $n_{f}$ are the resistance coefficient and flow exponent in the head loss formula respectively; $Q p_{i j}$ is the flow rate in pipe $i j . \mathbf{A}_{12}$ and $\mathbf{A}_{10}$ are the incidence matrices relating the pipes to the nodes with unknown and known heads, respectively. The elements of the incidence matrices are: -1 for pipe flows away from a node, +1 for pipe flows towards a node and 0 for pipes that are not connected to the node under consideration. $\mathbf{A}_{21}=\left(\mathbf{A}_{12}\right)^{\mathrm{T}}$ is the transpose of $\mathbf{A}_{12}$. $\mathbf{Q p}$ is the column vector of the unknown pipe flow rates. $\mathbf{H n}$ and $\mathbf{H}_{0}$ are column vectors for the unknown and known nodal heads, respectively. $\boldsymbol{Q} \boldsymbol{n}^{\boldsymbol{r e q}}$ is the column vector for the required nodal supplies.

To incorporate the nodal head-flow function, Eq. 5 is re-formulated as

$$
\left[\begin{array}{ccc}
\boldsymbol{A}_{11} & \vdots & \boldsymbol{A}_{12} \\
\cdots & \cdots & \cdots \\
\boldsymbol{A}_{21} & \vdots & \boldsymbol{A}_{22}
\end{array}\right]\left[\begin{array}{c}
\boldsymbol{Q p} \\
\cdots \\
\boldsymbol{H n}
\end{array}\right]=\left[\begin{array}{c}
-\boldsymbol{A}_{10} \boldsymbol{H}_{0} \\
\cdots \\
0
\end{array}\right]
$$

$\boldsymbol{A}_{22}$ is a diagonal matrix with elements $Q n_{i}\left(H n_{i}\right) / H n_{i} ; Q n_{i}\left(H n_{i}\right)$ is the nodal head-flow function in Eq. 1. Thus, Eq. 6 may be rearranged as.

$$
-\left[\begin{array}{ccc}
\boldsymbol{A}_{11} & \vdots & \boldsymbol{A}_{12} \\
\cdots & \cdots & \cdots \\
\boldsymbol{A}_{21} & \vdots & \boldsymbol{A}_{22}
\end{array}\right]\left[\begin{array}{c}
\boldsymbol{Q p} \\
\cdots \\
\boldsymbol{H n}^{k}
\end{array}\right]+\left[\begin{array}{c}
-\boldsymbol{A}_{10} \boldsymbol{H}_{0} \\
\cdots \\
0
\end{array}\right]=-\left[\begin{array}{c}
\boldsymbol{f}_{1} \\
\cdots \\
\boldsymbol{f}_{2}
\end{array}\right]
$$

where the vectors $\mathbf{f}_{1}=\mathbf{f}_{1}(\mathbf{Q n}, \mathbf{H n})$ and $\mathbf{f}_{2}=\mathbf{f}_{2}(\mathbf{Q n}, \mathbf{H n})$ indicate the errors, for any given approximate solution $(\mathbf{Q n}, \mathbf{H n})^{\mathrm{T}}$; at the solution, both $\mathbf{f}_{1}$ and $\mathbf{f}_{2}$ should be zero.

Considering Eq. 7, Eq. 6 may be solved by successive linearization using a first order Taylor series expansion that gives

$$
\left[\begin{array}{ccc}
\boldsymbol{D}_{11} & \vdots & \boldsymbol{A}_{12} \\
\cdots & \cdots & \cdots \\
\boldsymbol{A}_{21} & \vdots & \boldsymbol{D}_{22}
\end{array}\right]\left[\begin{array}{c}
\boldsymbol{Q}^{k+1}-\boldsymbol{Q} \boldsymbol{p}^{k} \\
\cdots \\
\boldsymbol{H} \boldsymbol{n}^{k+1}-\boldsymbol{H n}^{k}
\end{array}\right]=-\left[\begin{array}{ccc}
\boldsymbol{A}_{11} & \vdots & \boldsymbol{A}_{12} \\
\cdots & \cdots & \cdots \\
\boldsymbol{A}_{21} & \vdots & \boldsymbol{A}_{22}
\end{array}\right]\left[\begin{array}{c}
\boldsymbol{Q} \boldsymbol{p}^{k} \\
\cdots \\
\boldsymbol{H n}^{k}
\end{array}\right]+\left[\begin{array}{c}
-\boldsymbol{A}_{10} \boldsymbol{H}_{0} \\
\cdots \\
0
\end{array}\right]
$$

$\boldsymbol{D}_{11}$ is a diagonal matrix of the derivatives of the pipe head losses with respect to the pipe flow rates, whose elements are $n K_{i j}\left(Q p_{i j}\right)^{n_{f}-1} ; \boldsymbol{D}_{22}$ is a diagonal matrix of the derivatives of the nodal head-flow function with respect to the nodal heads, whose elements are described in Eq. $2 ; k$ is the iteration number. Eq. (8) gives the following routine for computing the nodal heads and pipe flows iteratively.

$$
\begin{gathered}
\boldsymbol{H} \boldsymbol{n}^{k+1}=\boldsymbol{A}^{-1} \boldsymbol{F} \\
\boldsymbol{Q} \boldsymbol{p}^{k+1}=\boldsymbol{Q} \boldsymbol{p}^{k}-\boldsymbol{D}_{11}^{-1}\left(\boldsymbol{A}_{11} \boldsymbol{Q} \boldsymbol{p}^{k}+\boldsymbol{A}_{12} \boldsymbol{H} \boldsymbol{n}^{k+1}+\boldsymbol{A}_{10} \boldsymbol{H}_{0}\right)
\end{gathered}
$$

In the above equations, i.e. Eq. 9 and 10,

$$
\boldsymbol{A}=\boldsymbol{A}_{21} \boldsymbol{D}_{11}^{-1} \boldsymbol{A}_{12}-\boldsymbol{D}_{22}
$$




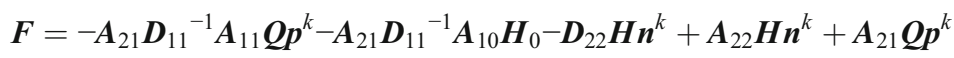

The procedure described above is for steady-state analysis. Extended period simulation may be carried out as described in Rossman (2000) and Siew and Tanyimboh (2010).

EPANET-PDX utilizes line minimization (Dennis and Schnabel 1996; Press et al. 2007) to help ensure global convergence in the computational solution of Eqs. 7 and 8. However, in an attempt to exploit the excellent computational properties of EPANET 2, the line minimization was not optimized in Siew and Tanyimboh (2012a). While the applications to date suggest that the strategy works well in general (Seyoum and Tanyimboh 2014; Siew et al. 2014, 2016), relatively poor and inconsistent performance was discovered subsequently, under conditions of extremely low pressure (Seyoum 2015). For the networks investigated, this corresponds roughly to a demand satisfaction of less than around $10 \%$.

The algorithm proposed herein allows unimpeded application of the line minimization procedure and, as a result, the computational efficiency is more consistent under all operating conditions. Overall, the number of minor iterations (i.e. iterations of the line minimization algorithm) has increased substantially while the number of major iteration (i.e. iterations of the global gradient algorithm) has decreased.

\section{Details of The Computational Solution Procedure}

The equations for conservation of energy and mass in Eq. 7 are:

$$
\begin{gathered}
\boldsymbol{f}_{1}(\boldsymbol{H} \boldsymbol{n}, \boldsymbol{Q p})=\boldsymbol{A}_{11} \boldsymbol{Q} \boldsymbol{p}^{k}+\boldsymbol{A}_{12} \boldsymbol{H} \boldsymbol{n}^{k}+\boldsymbol{A}_{10} \boldsymbol{H}_{0} \\
\boldsymbol{f}_{2}(\boldsymbol{H n}, \boldsymbol{Q} \boldsymbol{p})=\boldsymbol{A}_{21} \boldsymbol{Q} \boldsymbol{p}^{k}+\boldsymbol{A}_{22} \boldsymbol{H} \boldsymbol{n}^{k}
\end{gathered}
$$

Together, Eqs. 13 and 14 are a system of simultaneous non-linear equations, i.e. $\mathbf{f}=\mathbf{f}(\mathbf{H n}$, $\mathbf{Q p})=\left(\mathbf{f}_{\mathbf{1}}, \mathbf{f}_{\mathbf{2}}\right)^{\mathrm{T}}$, the solution of which is required. The aim of the line minimization procedure is to find an appropriate fraction $\lambda$ of the Newton step $\delta=(\delta \mathbf{H n}, \delta \mathbf{Q p})^{\mathrm{T}}$ that decreases the function $g$ in Eq. 15 sufficiently.

$$
g=\frac{1}{2} f \cdot f
$$

where $\mathbf{f . f}=\mathbf{f}^{\mathrm{T}} \mathbf{f}$ is the 2-norm of the vector of the system of equations $\mathbf{f} ; \delta \mathbf{H n}$ and $\delta \mathbf{Q p}$ are the vectors of the changes in the nodal heads and pipe flow rates, respectively. Given the scaler $\lambda$, the nodal heads Hn are updated iteratively as

$$
\boldsymbol{H} \boldsymbol{n}^{k+1}=\boldsymbol{H} \boldsymbol{n}^{k}+\lambda . \boldsymbol{\delta} \boldsymbol{H n} ; 0<\lambda \leq 1
$$

where $k$ is the iteration number and $\delta \mathbf{H n}$ is the Newton step for the nodal heads. The pipe flow rates $\boldsymbol{Q} \boldsymbol{p}^{k+1}$ are then updated by substituting the newly obtained nodal heads $\boldsymbol{H} \boldsymbol{n}^{k+1}$ into Eq. (10).

In each iteration of the global gradient algorithm, the full Newton step $\delta$ is attempted first. If the Newton step does not reduce the value of the function sufficiently, then backtracking along the Newton direction (i.e. the direction described by the elements of $\delta$ ), aimed at obtaining a better approximate solution of $\boldsymbol{f}(\boldsymbol{H n}, \boldsymbol{Q p})=0$, is carried out. The acceptance criterion for the new iterate is (Press et al. 2007) 


$$
g\left(\boldsymbol{H} \boldsymbol{n}^{k+1}, \boldsymbol{Q} \boldsymbol{p}^{k+1}\right) \leq g\left(\boldsymbol{H} \boldsymbol{n}^{k}, \boldsymbol{Q} \boldsymbol{p}^{k}\right)+\phi . \nabla g . \boldsymbol{\delta} ; 0<\phi<1
$$

where $\phi=10^{-4}$ (Dennis and Schnabel 1996; Press et al. 2007). $\nabla g$ is the gradient of $g(\boldsymbol{H n}$, $\boldsymbol{Q p})$ and $\boldsymbol{\delta}=(\boldsymbol{\delta} \boldsymbol{H} \boldsymbol{n}, \boldsymbol{\delta} \boldsymbol{Q p})^{T}$ is the Newton step and direction. Thus, $\nabla g . \boldsymbol{\delta}$ is the directional derivative or the rate of change of $g(\mathbf{H n}, \mathbf{Q p})$ in the Newton direction $\delta$. The initial rate of decrease of $g(\mathbf{H n}, \mathbf{Q p})$ is given in Press et al. (2007) as

$$
\nabla g . \boldsymbol{\delta}=(\boldsymbol{f} . \boldsymbol{J}) \cdot\left(-\boldsymbol{J}^{-1} \cdot \boldsymbol{f}\right)=-\boldsymbol{f} \cdot \boldsymbol{f}
$$

where $\mathbf{J}$ is the Jacobian matrix that comprises the first partial derivatives of $\boldsymbol{f}=\boldsymbol{f}(\boldsymbol{H n}, \boldsymbol{Q p})$ with respect to the nodal heads $H n_{i}, \forall i$, and pipe flow rates $Q p_{i j}, \forall i j$.

Backtracking is carried out if $g\left(\boldsymbol{H} \boldsymbol{n}^{k+1}, \boldsymbol{Q} \boldsymbol{p}^{k+1}\right)$ fails to meet the acceptance criterion in Eq. (17). In the backtracking procedure, application of the updating scheme in Eq. (16) (i.e. $\left.\boldsymbol{H n}^{k+1}=\boldsymbol{H} \boldsymbol{n}^{k}+\lambda . \delta \boldsymbol{H n} ; 0<\lambda \leq 1\right)$ converts $g\left(\mathbf{H n}^{\mathrm{k}+1}, \mathbf{Q p}^{\mathrm{k}+1}\right)$ into a function of $\lambda$ only, $g(\lambda)$. Backtracking seeks the value of $\lambda$ that minimizes $g(\lambda)$ and is accomplished by line minimization. In the first iteration of the line minimization, $g(\lambda)$ is modelled as a quadratic function. If any additional iterations are required then $g(\lambda)$ is modelled as a cubic function. The iterations continue until either Eq. (17) is satisfied or $\lambda$ reaches a specified minimum allowable value $\lambda_{\min }$. Dennis and Schnabel (1996) and Press et al. (2007) employed $\lambda_{\min }=0.1$ while Siew and Tanyimboh (2012a) used $\lambda_{\min }=0.2$ to avoid excessively small steps. $\lambda_{\min }=0.2$ was adopted herein. Details of the quadratic and cubic approximations of $g(\lambda)$ and its solution are available in Press et al. (2007).

The flow chart in Fig. 1 shows the integration of the line minimization in the global gradient algorithm. The global gradient algorithm starts with initial estimates of the nodal heads and pipe flow rates. The nodal elevations are taken as the initial nodal heads while the initial pipe flow rates are derived from an assumed velocity of $1 \mathrm{ft} . / \mathrm{s}(0.3048 \mathrm{~m} / \mathrm{s})$. In each iteration of the global gradient algorithm, the Euclidean norm of the energy and mass balance equations is checked.

It was observed that, in some cases, when $\lambda$ reached $\lambda_{\min }=0.2$, the norm failed to reduce in consecutive iterations. If this situation arises, the algorithm calculates a new Newton step i.e. $\delta=(\delta \boldsymbol{H n}, \boldsymbol{\delta} \boldsymbol{Q p})^{T}$ as a major iteration. Before doing so, however, the nodal heads are updated first with $\lambda=\lambda_{\min }=0.2$ in Eq. 16 as shown Fig. 1. This additional measure was introduced to help prevent premature convergence as it enables the major iterations to resume from a different point by updating $\mathbf{H n}$ without updating $\mathbf{Q p}$ at the same time. In the investigations herein, though rare, without this extra measure, there were instances of spurious convergence. False convergence may occur if a local minimum of Eq. $15, g=\frac{1}{2} \boldsymbol{f} . \boldsymbol{f}$, is found that is not a root of Eq. 7, i.e. $\boldsymbol{f}=\boldsymbol{f}(\boldsymbol{H n}, \boldsymbol{Q p})=0$ (Press et al. 2007).

The iterations of the global gradient algorithm continue until the convergence criteria are fulfilled. The EPANET-PDX convergence criteria of $0.001 \mathrm{ft}$. $\left(3.048 \times 10^{-4} \mathrm{~m}\right)$ for the maximum change in the nodal heads and 0.001 cubic feet per second $\left(2.832 \times 10^{-5} \mathrm{~m}^{3} / \mathrm{s}\right)$ for the maximum change in the pipe flow rates between successive iterations (Siew and Tanyimboh 2012a) were retained herein. The EPANET-PDX software was developed by upgrading the source code of EPANET 2.

\section{Results and Discussion}

Three examples are provided to illustrate the properties of the proposed algorithm. The first two are networks taken from literature, mainly to illustrate the performance in terms of the 


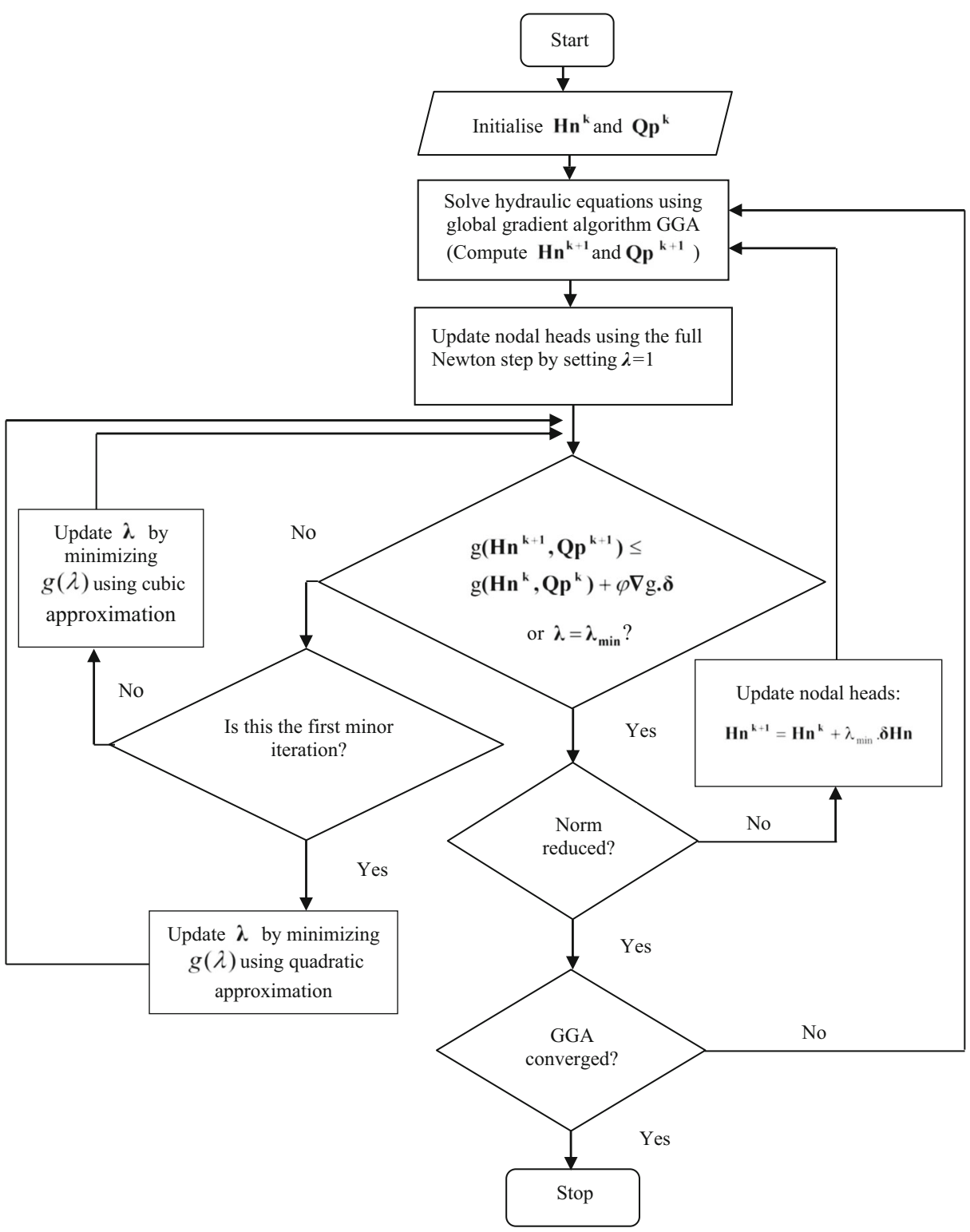

Fig. 1 Integration of line minimization in the global gradient algorithm (GGA)

number of iterations and CPU time required for convergence. The third example demonstrates the effectiveness on a real-world network, based on extended period simulation under various operating conditions including extremely low pressure.

All the simulations were carried out on an Intel Xeon workstation (with two processors of CPU $2.4 \mathrm{GHz}$ and RAM of $16 \mathrm{~GB}$ ). For simplicity hereafter, the new algorithm is called EPANET-PDX (0.2) while the original EPANET-PDX is called EPANET-PDX (0.1). In all the simulations, the assumed head below which nodal flow is zero was taken as the nodal elevation. In all the examples considered, the previous and new algorithms yielded essentially 
identical solutions (i.e. nodal flows, nodal heads and pipe flows) while the latter required fewer, or the same number of, iterations of the global gradient algorithm, as illustrated in the Appendix (i.e. online supplementary data).

\subsection{Steady State Analysis of Small Networks}

Two examples were considered for illustration purposes, i.e. the six-loop and Anytown networks in Fig. 2. The first example relates to the network in Fig. 2a (Todini 2003) that has one source, 11 demand nodes, 17 pipes of length $500 \mathrm{~m}$ and Hazen-Williams roughness coefficient of 130. The elevations of the nodes are: $80 \mathrm{~m}$ for nodes 3 and $10 ; 85 \mathrm{~m}$ for nodes 4 , 5,7 and 9; $90 \mathrm{~m}$ for nodes 2, 6, 8 and 11 ; and $100 \mathrm{~m}$ for node 12 . The demands in $\mathrm{m}^{3} / \mathrm{s}$ are: 0.01 for nodes 8 and $12 ; 0.04$ for nodes 4,9 and 11 ; and 0.05 for nodes 2, 5-7 and 10. The pipe diameters in mm are: 100 for pipes $7,10,13,14,16$ and 17; 150 for pipes $3,6,9,12$ and 15; 200 for pipes 2, 5, 8 and 11; and 250 for pipes 1 and 4 .

Steady-state simulations were performed as follows. (a) Simulations with assumed required residual pressures of $30 \mathrm{~m}, 20 \mathrm{~m}$ and $10 \mathrm{~m}$ at all the demand nodes with the source head fixed at $150 \mathrm{~m}$. (b) Simulations with source heads of $150 \mathrm{~m}, 125 \mathrm{~m}, 100 \mathrm{~m}$ and $75 \mathrm{~m}$ with an assumed required residual pressure of $30 \mathrm{~m}$ at all the demand nodes. Table 1 summarises the computational performances of EPANET-PDX (0.1) and (0.2). Generally EPANET-PDX (0.2) required fewer iterations while the CPU times of both models were comparable. The nodal heads and flows of both models were essentially identical.

The second example is based on the "Anytown" network (Fig. 2b) used previously as a benchmark optimisation problem (Walski et al. 1987). Siew and Tanyimboh (2012a) modified the system and operational data to create a pressure-deficient condition. They set the diameter of pipes $10,13,14,15,16$ and 25 as $0.3048 \mathrm{~m}$ (12 in); the diameters of the six pipes mentioned were to be determined in the optimization problem (Walski et al. 1987). Also, the demands at nodes 2, 4, 5, 9, 10, 12 and 15 were taken as $3.155 \mathrm{l} / \mathrm{s}$. Additional data for the pipes and nodes are available in Walski et al. (1987). A water treatment plant supplies the network, with three identical pumps that operate in parallel. The water level in the treatment plant is fixed at $3.05 \mathrm{~m}(10 \mathrm{ft})$. The required pressure for all the demand nodes is $28.12 \mathrm{~m}$ ( $40 \mathrm{psi}$ ). The network has two storage tanks (Tanks 41 and 42). Herein, the pipes that connect the tanks to the network were closed.

Using the base demands, and taking the demand factors (i.e. the nodal demand multipliers) as 1.0 , a steady-state simulation of the modified network with the tanks closed showed there was insufficient pressure, as only $87 \%$ of the total demand was satisfied. Comparisons of the nodal heads and outflows for all the nodes showed that both EPANET-PDX (0.1) and (0.2) yielded essentially identical results. The number of iterations required by EPANET-PDX (0.1) and $(0.2)$ to converge was 13 and 10 , respectively. Both models required on average $0.04 \mathrm{~s}$ to complete the simulation.

\subsection{Extended Period Simulation of Real World Network}

An investigation was conducted based on a network that is a hydraulic demand zone (referred to hereafter as a water supply zone) in the UK. Figure 2c shows the network that comprises 251 pipes of various lengths, 228 demand nodes (including the fire hydrants), 3 demand categories and 29 fire hydrants; the pipe diameters range from $32 \mathrm{~mm}$ to $400 \mathrm{~mm}$. The network obtains water entirely from the neighbouring water supply zones through five variable-head supply nodes (i.e. nodes R1-R5 in Fig. 2c). Details of the temporal variations in the demands 


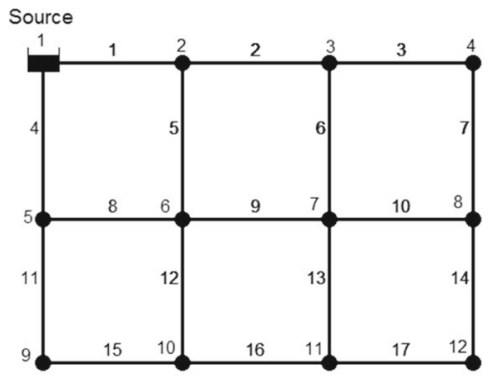

(a) Example 1

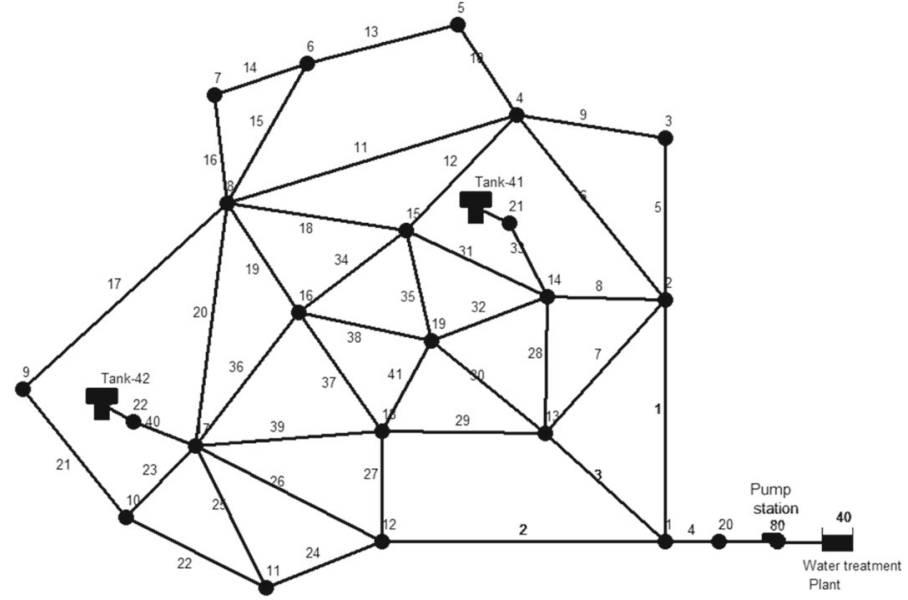

(b) The Anytown network in Example 2

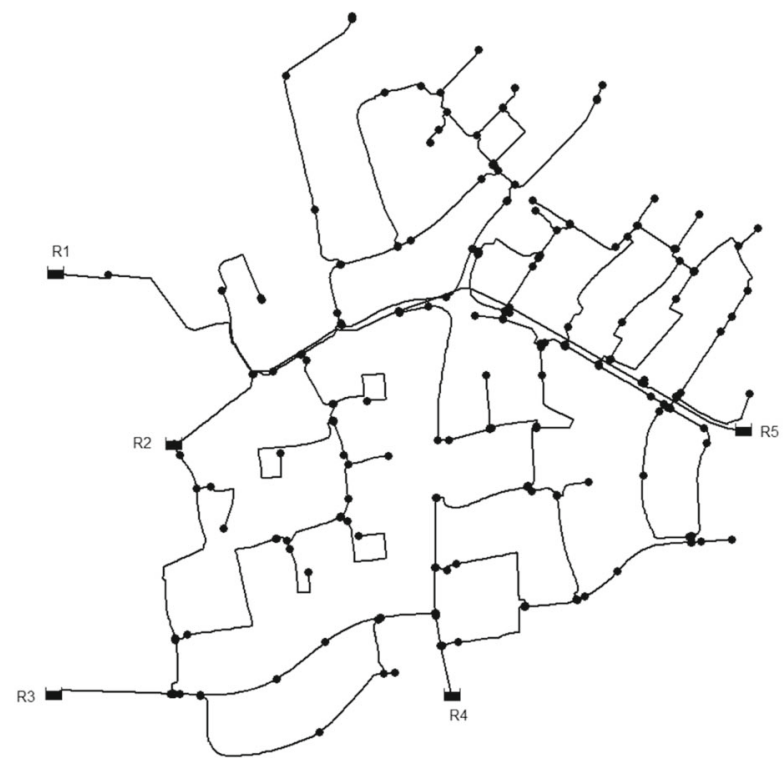

(c) Example 3

Fig. 2 Topologies of the networks investigated 
and supply-node heads are available in Seyoum and Tanyimboh (2014). The range of variation in the heads at the supply nodes is insignificant. Therefore, here, to simplify the interpretation of the results, the supply nodes were modelled as constant-head nodes with water levels of $155 \mathrm{~m}$ each.

The water utility provided the network and dynamic operational data from a geographical information system database, and a calibrated EPANET model. The demand categories comprise domestic and 10-h commercial demands, and unaccounted for water (Kanakoudis and Gonelas 2016). There are 29 different fire demands of one hour each at the respective fire hydrants. The head loss in the pipes due to friction is derived from the Darcy-Weisbach formula (Rossman 2000). The required residual head at all the demand nodes is $20 \mathrm{~m}$.

All the analyses reported on this network consist of extended period simulations of $31 \mathrm{~h}$, with a hydraulic time step of one hour. For each of the three simulation models considered, i.e. EPANET 2, EPANET-PDX (0.1) and (0.2), 66 extended period simulations were carried out in total, for the normal and pressure-deficient conditions. The simulations included pipe closures (10 cases) and varying head levels at the supply nodes (56 cases).

\subsubsection{Source Head Variations}

The investigation covered all levels of demand satisfaction from zero to $100 \%$. Supply node heads from $75 \mathrm{~m}$ to $130 \mathrm{~m}$ in steps of $1 \mathrm{~m}$ were considered. Each model executed the 56 simulations without convergence failures. EPANET-PDX (0.1) and (0.2) gave identical results of flow and pressure. The computational performance was assessed considering three scenarios, i.e. normal, low and extremely low pressure.

Normal pressure was taken as $99.9 \%$ demand satisfaction and above, for the network as a whole. This corresponds to heads at the supply nodes from approximately $116 \mathrm{~m}$ to $130 \mathrm{~m}$. In the extended period simulation, the number of major iterations required on average per steadystate simulation was 7.00, 5.00 and 5.16 for EPANET-PDX (0.1), EPANET-PDX (0.2) and EPANET 2, respectively. Figure 3a compares the CPU times. On average, EPANET-PDX (0.1) and $(0.2)$ required about $0.27 \mathrm{~s}$ and $0.29 \mathrm{~s}$, respectively, per extended period simulation, compared to $0.15 \mathrm{~s}$ for EPANET 2.

Table 1 Major iterations and CPU times for the network in Example 1

\begin{tabular}{|c|c|c|c|c|c|c|c|}
\hline & \multirow{2}{*}{\multicolumn{3}{|c|}{$\begin{array}{l}\text { Variations in required nodal } \\
\text { residual heads } \\
\text { Required residual heads (m) }\end{array}$}} & \multicolumn{4}{|c|}{ Variations in reservoir heads } \\
\hline & & & & \multicolumn{4}{|c|}{ Reservoir heads (m) } \\
\hline & $30^{\mathrm{a}}(88 \%)$ & $20(89 \%)$ & $10(91 \%)$ & $150(88 \%)$ & $125(71 \%)$ & $100(29 \%)$ & $75(0.04 \%)$ \\
\hline \multicolumn{8}{|c|}{ Number of major iterations } \\
\hline EPANET-PDX (0.1) & 9 & 11 & 29 & 9 & 9 & 10 & 8 \\
\hline EPANET-PDX (0.2) & 9 & 10 & 16 & 9 & 9 & 7 & 7 \\
\hline \multicolumn{8}{|l|}{ CPU times (seconds) } \\
\hline EPANET-PDX (0.1) & 0.03 & 0.03 & 0.04 & 0.03 & 0.04 & 0.03 & 0.03 \\
\hline EPANET-PDX (0.2) & 0.05 & 0.04 & 0.04 & 0.05 & 0.03 & 0.03 & 0.03 \\
\hline
\end{tabular}

\footnotetext{
${ }^{\mathrm{a}}$ The values in parentheses are the percentages of the total network demand satisfied
} 
EPANET 2, in general, seemed more efficient and consistent. It is worth emphasizing, however, that EPANET 2 and EPANET-PDX apply convergence criteria that are different (Siew and Tanyimboh 2012a). The default criterion used in EPANET 2 is that the ratio of the sum of the absolute values of the changes in the pipe flow rates to the total flow in all the pipes should be less than 0.001 . Therefore, it is worth emphasizing also that the EPANET 2 results serve as a reference rather than a direct comparison. The CPU times refer to extended period simulations of $31 \mathrm{~h}$ with a hydraulic time step of one hour. Burger et al. (2015) compared different demand-driven analysis solvers to EPANET 2 and concluded that EPANET 2 was the fastest for practical applications.

Fig. 3 CPU times as a function of pressure in the network in Example 3. DSR is the demand satisfaction ratio, i.e. the ratio of the available flow to the required flow

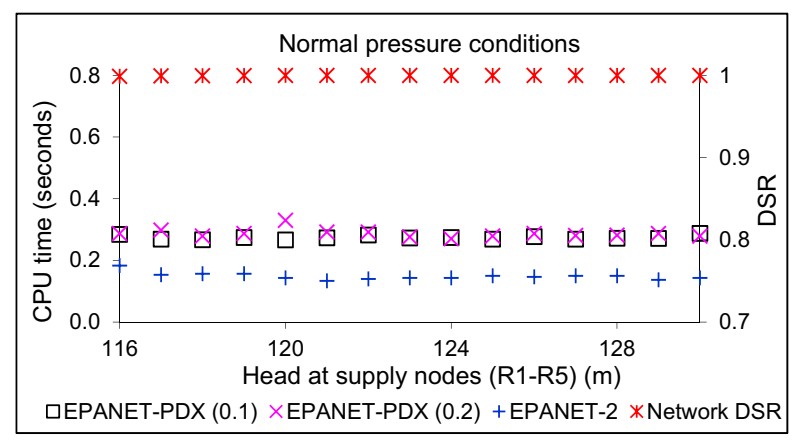

(a) Normal pressure conditions

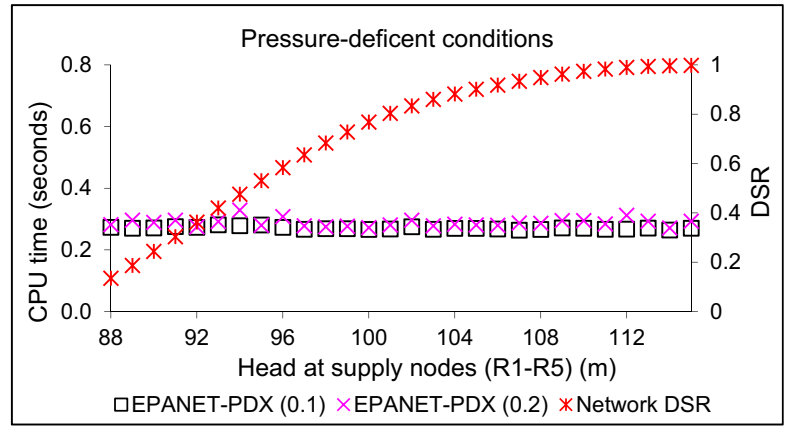

(b) Pressure-deficient conditions

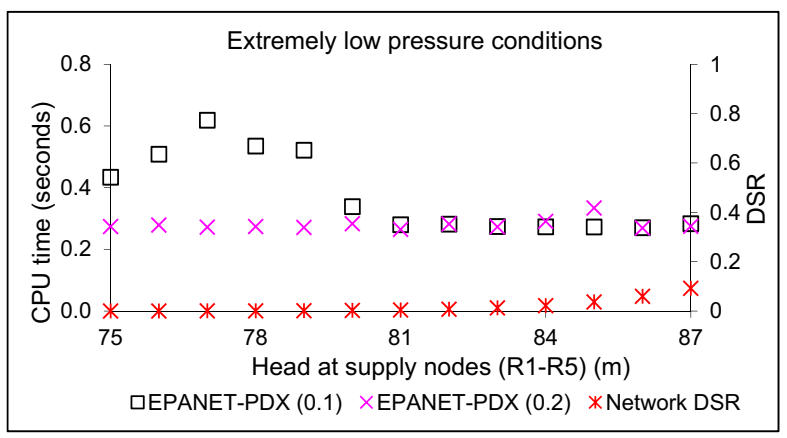

(c) Extremely low pressure conditions 
The pressure-deficient condition was assumed to include demand satisfaction of $9.23 \%$ to $99.9 \%$, i.e. from just below satisfactory pressure to just above extremely low-pressure. The corresponding heads at the supply nodes were $88 \mathrm{~m}$ to $115 \mathrm{~m}$ approximately. EPANET 2 results are not included in this scenario and the next, as it is not suitable for operating conditions with insufficient pressure. The major iterations and CPU times were similar to the normal operating condition. On average, the number of iterations per steady state simulation was 7.00 and 5.04 for EPANET-PDX (0.1) and EPANET-PDX (0.2), respectively. The CPU times were comparable, i.e. $0.27 \mathrm{~s}$ and $0.29 \mathrm{~s}$ for EPANET-PDX $(0.1)$ and EPANET-PDX (0.2) respectively, per extended-period simulation (Fig. 3b).

The performance under conditions of extremely low pressure is important from the perspective of evolutionary optimisation algorithms that may generate extremely infeasible solutions. Recent results have demonstrated the advantages of retaining nondominated infeasible solutions until the end of the optimization (Siew and Tanyimboh 2012b; Eskandar et al. 2012; Saleh and Tanyimboh 2013, 2014, 2016; Siew et al. 2014, 2016; Tanyimboh and Seyoum 2016) as infeasible solutions usually contain useful genetic materials (Herrera et al. 1998).

Supply node heads from $75 \mathrm{~m}$ to $87 \mathrm{~m}$, approximately, with network demand satisfaction below $9.23 \%$, were deemed extremely low (Fig. 3c). EPANET-PDX (0.2) achieved significant improvements in both the number of iterations and CPU time. The average number of iterations was 6.85 and 4.08 for EPANET-PDX (0.1) and EPANET-PDX (0.2), respectively. The average CPU time was $0.38 \mathrm{~s}$ and $0.28 \mathrm{~s}$ for EPANET-PDX (0.1) and EPANET-PDX (0.2), respectively. For supply node heads from $75 \mathrm{~m}$ to $81 \mathrm{~m}$, EPANET-PDX (0.2) was up to around $50 \%$ faster.

\subsubsection{Pipe Closures}

In this scenario, the pipes from three supply nodes out of five (R1-R5) were closed. In total, 10 cases were considered and it was observed that the nodal demands were fully satisfied in each case. Table 2 provides a summary of the results. On average, there were 6.77, 5.22 and 4.90 iterations per steady state simulation for EPANET-PDX (0.1), EPANET-PDX (0.2) and

Table 2 Major iterations and CPU times for the pipe closures in Example 3

\begin{tabular}{|c|c|c|c|c|c|c|}
\hline \multirow[t]{2}{*}{$\begin{array}{l}\text { Closed Supply } \\
\text { Nodes }\end{array}$} & \multicolumn{3}{|c|}{$\begin{array}{l}\text { Mean number of major iterations per } \\
\text { steady-state simulation }\end{array}$} & \multicolumn{3}{|c|}{$\begin{array}{l}\text { CPU times for extended period simulations } \\
\text { (seconds) }\end{array}$} \\
\hline & $\begin{array}{l}\text { EPANET-PDX } \\
(0.1)\end{array}$ & $\begin{array}{l}\text { EPANET-PDX } \\
(0.2)\end{array}$ & EPANET 2 & $\begin{array}{l}\text { EPANET-PDX } \\
(0.1)\end{array}$ & $\begin{array}{l}\text { EPANET-PDX } \\
(0.2)\end{array}$ & EPANET 2 \\
\hline $\mathrm{R} 1,2,3$ & 6.969 & 5.250 & 4.875 & 0.197 & 0.202 & 0.137 \\
\hline $\mathrm{R} 1,2,4$ & 6.719 & 5.156 & 5.000 & 0.200 & 0.209 & 0.137 \\
\hline $\mathrm{R} 1,2,5$ & 6.781 & 5.250 & 4.875 & 0.193 & 0.207 & 0.140 \\
\hline $\mathrm{R} 1,3,4$ & 6.750 & 5.250 & 4.875 & 0.210 & 0.238 & 0.140 \\
\hline $\mathrm{R} 1,3,5$ & 6.563 & 5.188 & 4.844 & 0.197 & 0.202 & 0.147 \\
\hline $\mathrm{R} 1,4,5$ & 6.719 & 5.313 & 4.875 & 0.190 & 0.204 & 0.147 \\
\hline $\mathrm{R} 2,3,4$ & 6.781 & 5.125 & 4.844 & 0.200 & 0.205 & 0.147 \\
\hline $\mathrm{R} 2,3,5$ & 6.969 & 5.250 & 4.938 & 0.197 & 0.197 & 0.137 \\
\hline $\mathrm{R} 2,4,5$ & 6.656 & 5.125 & 4.969 & 0.200 & 0.202 & 0.140 \\
\hline $\mathrm{R} 3,4,5$ & 6.750 & 5.250 & 4.875 & 0.200 & 0.209 & 0.153 \\
\hline
\end{tabular}


EPANET 2, respectively. The corresponding average CPU times were $0.20,0.21$ and $0.14 \mathrm{~s}$, respectively, per extended period simulation.

\subsubsection{Effectiveness of the Line Minimization}

Figure $4 \mathrm{a}$ shows the number of minor iterations as a function of the pressure. In general, under conditions of extremely low pressure, EPANET-PDX (0.1) applied one minor iteration per steady state simulation while EPANET-PDX (0.2) applied an average of 10.30 . Figure $4 \mathrm{~b}$ shows the number of minor iterations for the supplynode closures. On average, EPANET-PDX (0.2) performed 10.43 minor iterations per steady state simulation whereas EPANET-PDX (0.1) performed only 0.16 . Overall, it seems that the line minimization in EPANET-PDX (0.1) served mainly as a safety net to avoid divergence of the major iterations.

Figure 5 shows the norm of the constitutive equations at successive iterations, for three typical extended period simulations. Each simulation comprises 31 steady state simulations. It is worth mentioning that the norm in Fig. 5 is based on cubic feet per second and feet, for flow continuity and head loss, respectively. In SI units $\left(\mathrm{m}^{3} \mathrm{~s}^{-1}\right.$ and $\left.\mathrm{m}\right)$, the values would be much smaller.

It was observed that for the network demand satisfaction of $0.01 \%$ (Fig. 5c), the norm increased at the second iteration before decreasing consistently in subsequent iterations. Dennis and Schnabel (1996, pp. 129) discuss the circumstances in which the line minimization may loop indefinitely. This may arise if the Newton direction is not a descent direction. It may

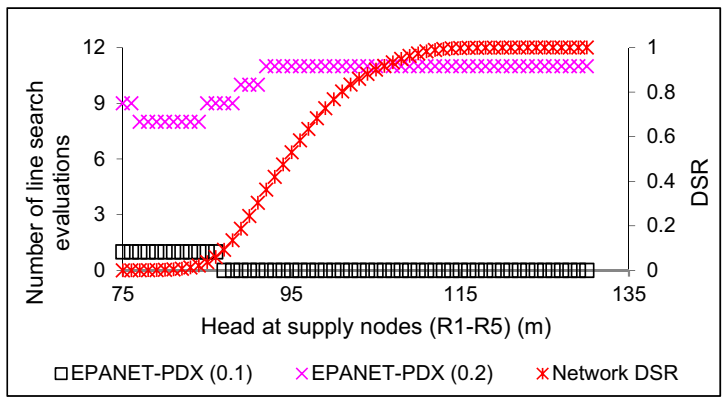

(a) Mean number of minor iterations per steady state simulation as a function of pressure

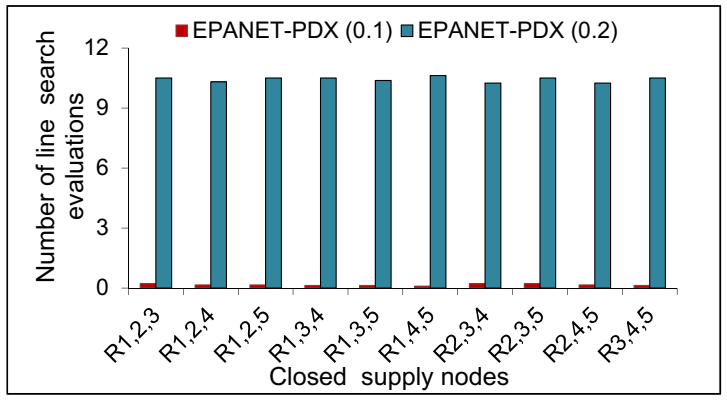

(b) Mean number of minor iterations per steady state simulation for pipe closures

Fig. 4 Comparison of the minor iterations based on the network in Example 3 
also be due to spurious convergence. The procedure proposed herein handles an increase in the norm by updating the nodal heads using a default minimum step length in the Newton direction that allows the algorithm to continue (Section 3).

\section{Conclusions}

An approach for integrating a line minimization procedure in the global gradient algorithm for pressure-driven modelling of water distribution networks is proposed. The algorithm's

Fig. 5 The norm of the system of equations. Each extended period simulation shown comprises 31 steady state simulations of the network in Example 3

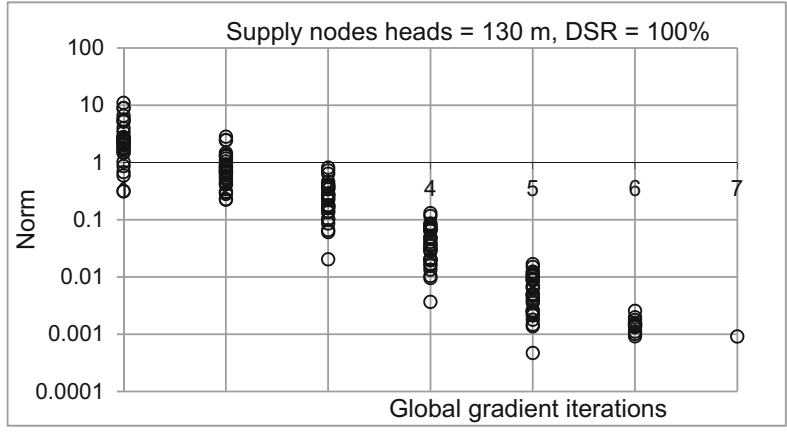

(a) Demand satisfaction of $100 \%$

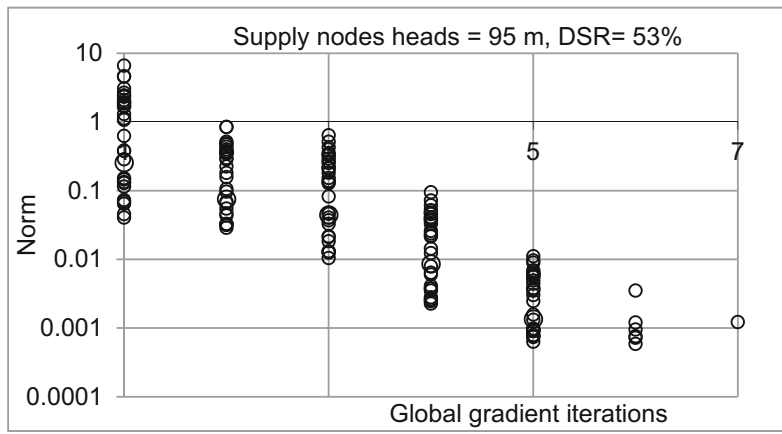

(b) Demand satisfaction of 53\%

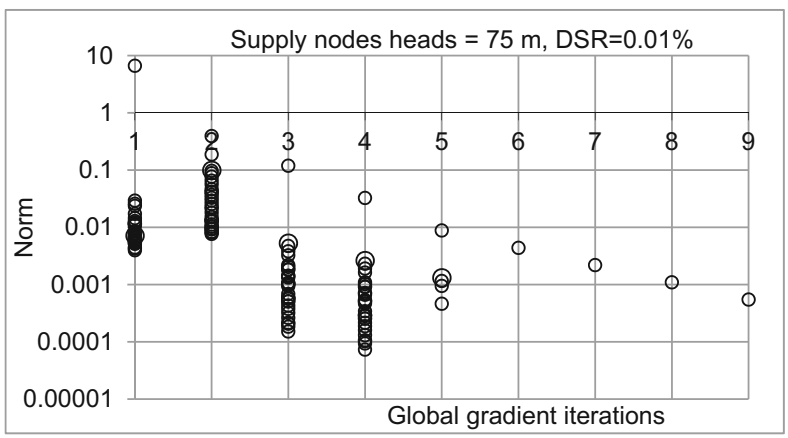

(c) Demand satisfaction of $0.01 \%$ 
performance was satisfactory when simulating two networks in the literature and a real-world network. For simulations based on the real-world network, the algorithm achieved significant improvements in both the number of major iterations ( $40 \%$ ) and CPU time (26\%), in conditions of extremely low pressure, compared to Siew and Tanyimboh (2012a). In particular, for supply node heads from $75 \mathrm{~m}$ to $81 \mathrm{~m}$, the CPU time was reduced by up to $50 \%$ approximately. It may be noted also, that the hydraulic and water quality modelling functionality of EPANET 2 has been preserved.

The algorithm proposed allows unimpeded application of the line minimization procedure and, as a result, the computational properties are more consistent under all operating conditions. Overall, the number of minor iterations (i.e. iterations of the line minimization algorithm) has increased substantially while the number of major iteration (i.e. iterations of the global gradient algorithm) has decreased. Where direct comparisons with EPANET 2 were possible, i.e. for operating conditions with satisfactory pressure, EPANET 2 was consistently faster.

Acknowledgment This project was funded in part by the UK Engineering and Physical Sciences Research Council (EPSRC Grant Reference EP/G055564/1), the British Government (Overseas Research Students Awards Scheme) and the University of Strathclyde. This financial support is acknowledged with thanks. The authors also thank Dr. Lewis Rossman of the United States Environmental Protection Agency, for assisting with the source code of the EPANET 2 computer program, and Veolia Water UK (now Affinity Water) for the support they provided.

\section{Compliance with Ethical Standards}

Conflict of Interest There is no conflict of interest.

Open Access This article is distributed under the terms of the Creative Commons Attribution 4.0 International License (http://creativecommons.org/licenses/by/4.0/), which permits unrestricted use, distribution, and reproduction in any medium, provided you give appropriate credit to the original author(s) and the source, provide a link to the Creative Commons license, and indicate if changes were made.

\section{References}

Abdy Sayyed MAH, Gupta R, Tanyimboh TT (2015) Noniterative application of EPANET for pressure dependent modelling of water distribution systems. Water Resour Manag 29(9):3227-3242

Bragalli C, Fortini M, Todini E (2016) Enhancing knowledge in water distribution networks via data assimilation. Water Resour Manag 30(11):3689-3706

Burger G, Sitzenfrei R, Kleidorfer M, Rauch W (2015) Quest for a new solver for EPANET 2. Journal of Water Resource Planning and Management. doi:10.1061/(ASCE)WR.1943-5452.0000596

Ciaponi C, Franchioli L, Murari E, Papiri S (2015) Procedure for defining a pressure-outflow relationship regarding indoor demands in pressure-driven analysis of water distribution networks. Water Resour Manag 29(3):817-832

Dennis JE, Schnabel RB (1996) Numerical methods for unconstrained optimization and nonlinear equations. SIAM, Philadelphia

Deuerlein J, Simpson A, Dempe S (2009) Modeling the behavior of flow regulating devices in water distribution systems using constrained nonlinear programming. J Hydraul Eng 135(11):970-982

Dini M, Tabesh M (2014) A new method for simultaneous calibration of demand pattern and Hazen-Williams coefficients in water distribution systems. Water Resour Manag 28:2021-2034

Elhay S, Piller O, Deuerlein J, Simpson A (2015) A robust, rapidly convergent method that solves the water distribution equations for pressure-dependent models. Journal of Water Resource Planning and Management. doi:10.1061/(ASCE)WR.1943-5452.0000578

Eskandar H, Sadollah A, Bahreininejad A, et al. (2012) Water cycle algorithm - a novel metaheuristic optimization method for solving constrained engineering optimization problems. Comput Struct:110-111

Gorev NB, Kodzhespirova IF (2013) Noniterative implementation of pressure-dependent demands using the hydraulic analysis engine of EPANET 2. Water Resour Manag 27(10):3623-3630 
Herrera F, Lozano M, Verdegay JL (1998) Tackling real-coded genetic algorithms: operators and tools for behavioural analysis. Artif Intell Rev 12:265-319

Kanakoudis V, Gonelas K (2016) Analysis and calculation of the short and long run economic leakage level in a water distribution system. Water utility Journal 12:57-66

Kang D, Lansey K (2014) Novel approach to detecting pipe bursts in water distribution networks. J Water Resour Plan Manag 140(1):121-127

Kougias IP, Theodossiou NP (2013) Multi-objective pump scheduling optimization using harmony search algorithm and polyphonic HSA. Water Resour Manag 27(5):1249-1261

Kovalenko Y, Gorev NB, Kodzhespirova IF, Prokhorov E, Trapaga G (2014) Convergence of a hydraulic solver with pressure-dependent demands. Water Resour Manag 28(4):1013-1031

Kun D, Tian-Yu L, Jun-Hui W, Jin-Song G (2015) Inversion model of water distribution systems for nodal demand calibration. J. Water Resour. Plann. Manage. doi:10.1061/(ASCE)WR.1943-5452. 0000506

Laucelli D, Giustolisi O (2015) Vulnerability assessment of water distribution networks under seismic actions. J Water Resour Plann Manage. doi:10.1061/(ASCE)WR.1943-5452.0000478,04014082

Méndez M, Araya JA, Sanchez LD (2013) Automated parameter optimization of a water distribution system. J Hydroinf 15(1):71-85

Press WH, Teukolsky SA, Vetterling WT, Flannery BP (2007) Numerical recipes: The art of scientific computing. Press, Cambridge University

Ray T, Singh HK, Isaacs A, Smith W (2009) Infeasibility driven evolutionary algorithm for constrained optimization, in Constraint Handling in Evolutionary Optimization Studies in Computational Intelligence: Vol.198. Springer, Berlin, pp. 145-165

Rossman LA (2000) EPANET 2 Users manual. Water supply and water resources division. National Risk Management Research Laboratory, US EPA, Cincinnati

Rossman LA (2007) Discussion of "Solution for water distribution systems under pressure-deficient conditions" by Ang and Jowitt. Journal of Water Resource Planning and Management 133(6):566-567

Saleh SH, Tanyimboh TT (2013) Coupled topology and pipe size optimization of water distribution systems. Water Resour Manag 27(14):4795-4814

Saleh SH, Tanyimboh TT (2014) Optimal design of water distribution systems based on entropy and topology. Water Resour Manag 28(11):3555-3575

Saleh SHA, Tanyimboh TT (2016) Multi-directional maximum-entropy approach to the evolutionary design optimization of water distribution systems. Water Resour Manag 30(6):1885-1901

Seifollahi-Aghmiuni S, Bozorg Haddad O, Mariño MA (2013) Water distribution network risk analysis under simultaneous consumption and roughness uncertainties. Water Resour Manag 27(7):2595-2610

Seyoum AG (2015) Head dependent modelling and optimization of water distribution systems. PhD thesis, University of Strathclyde, Glasgow

Seyoum AG, Tanyimboh TT (2014) Pressure dependent network water quality modelling. Proceedings of ICE: Water Management 167(6):342-355

Siew C, Tanyimboh TT (2010) Pressure-dependent EPANET extension: Extended period simulation. 12th International Conference on Water Distribution Systems Analysis, Tucson, Arizona. doi:10.1061/ 41203(425) 10

Siew C, Tanyimboh TT (2012a) Pressure-dependent EPANET extension. Water Resour Manag 26(6): 1477-1498

Siew C, Tanyimboh TT (2012b) Penalty-free feasibility boundary convergent multi-objective evolutionary algorithm for the optimization of water distribution systems. Water Resour Manag 26(15): 4485-4507

Siew C, Tanyimboh TT, Seyoum AG (2014) Assessment of penalty-free multi-objective evolutionary optimization approach for the design and rehabilitation of water distribution systems. Water Resour Manag 28(2): 373-389

Siew C, Tanyimboh TT, Seyoum AG (2016) Penalty-free multi-objective evolutionary approach to optimization of Anytown water distribution network. Water Resour Manag 30(11):3671-3688

Singh HK, Isaacs A, Ray T, Smith W (2008) Infeasibility driven evolutionary algorithm (IDEA) for engineering design optimization. 21st Australiasian Joint Conference on. Artificial Intelligence AI-08:104-115

Sivakumar P, Prasad RK (2015) Extended period simulation of pressure-deficient networks using pressure reducing valves. Water Resour Manag 29(5):1713-1730

Spiliotis M, Tsakiris G (2011) Water distribution system analysis: Newton-Raphson method revisited. J Hydraul Eng ASCE 137(8):852-855

Tanyimboh TT, Seyoum AG (2016) Multiobjective evolutionary optimization of water distribution systems: Exploiting diversity with infeasible solutions. J Environ Manag 183:133-141. doi:10.1016/j.jenvman.2016. 08.048 
Tanyimboh TT, Templeman AB (2010) Seamless pressure deficient water distribution system model. Proceedings of ICE: Water Management 163(8):389-396

Tanyimboh TT, Tahar B, Templeman AB (2003) Pressure-driven modelling of water distribution systems. Water science and technology. Water Supply 3(1-2):255-261

Tao T, Huang H, Li F, Xin K (2014) Burst detection using an artificial immune network in water-distribution systems. J. Water Resour. Plann. Manage. doi:10.1061/(ASCE)WR.1943-5452.0000405,04014027

Todini, E. (2003) A more realistic approach to the extended period simulation of water distribution networks. Advances in Water Supply Management, Maksimovic, C., Butler, D. and Memon, F.A. (eds.), Balkema, The Netherlands, pp.173-184.

Todini E, Pilati S (1988) In: Coulbeck B, Chun-Hou O (eds) A gradient algorithm for the analysis of pipe networks. Computer applications in water supply: systems analysis and SIMULATION: Vol. 1. Research Studies Press, Taunton, pp. 1-20

Tsakiris G, Spiliotis M (2014) A Newton-Raphson analysis of urban water systems based on nodal head-driven outflow. European Journal of Environmental and Civil Engineering 18(8):882-896

Vairagade SA, Abdy Sayyed MAH, Gupta R (2015) Node head flow relationships in skeletonized water distribution networks for predicting performance under deficient conditions. World Environmental and Water Resources Congress, Austin, Texas

Walski TM, Brill ED, Gessler J, Goulter IC, Jeppson RM, Lansey K, Lee HL, Liebman JC, Mays L, Morgan DR, Ormsbee L (1987) Battle of the network models: epilogue. Journal of Water Resource Planning and Management 113(2):191-203

Yang X, Boccelli D (2014) Bayesian approach for real-time probabilistic contamination source identification. J. Water Resour. Plann. Manage. doi:10.1061/(ASCE)WR.1943-5452.0000381,04014019 